\title{
The long $\gamma$-ray burst rate and the correlation with host galaxy properties
}

\author{
J. Elliott ${ }^{\star a}$, J. Greiner ${ }^{a}$, S. Khochfar ${ }^{a}$, P. Schady ${ }^{a}$, J. L. Johnson ${ }^{a b}$, and A. Rau ${ }^{a}$ \\ ${ }^{a}$ Max-Planck-Institut für extraterrestrische Physik \\ Postfach 1312, Giessenbachstr., 85741 Garching, Germany \\ ${ }^{b}$ Los Alamos National Laboratory \\ Los Alamos, NM 87545, USA \\ E-mail: jonnyelliott@mpe.mpg.de, jcg@mpe.mpg.de, sadeghk@mpe.mpg.de, \\ pschady@mpe.mpg.de, jjohnson@mpe.mpg.de, arau@mpe.mpg.de
}

\begin{abstract}
The association of long gamma-ray bursts (LGRB) with the death of massive stars gives the prospect of utilising LGRBs to trace the high redshift cosmic star formation history (CSFH), competing or even surpassing conventional methods. However, a consensus on the manner in which the LGRB rate (LGRBR) traces the CSFH must be reached. Driven by recent highly complete LGRB samples, obtained by the Gamma-Ray burst Optical Near-infrared Detector (GROND) over the past 4 years, and new evidence of LGRBs occurring in more massive and metal rich galaxies than previously thought, the possible biases of the LGRBR-CSFH connection are investigated over a large range of galaxy properties. It is found that there is no strong preference for metallicity cuts or fixed galaxy mass boundaries and that there are no unknown redshift effects.
\end{abstract}

Gamma-Ray Bursts 2012 Conference-GRB2012,

May 07-11, 2012

Munich, Germany

* Speaker. 


\section{Introduction}

Achieving luminosities greater than $10^{52} \mathrm{erg} \mathrm{s}^{-1}$, long-duration gamma-ray bursts (LGRBs) are tied to some of the most catastrophic explosions in the universe. Their ultra luminous nature and simple power law spectrum makes LGRBs potentially great probes for the high redshift Universe. The connection of LGRBs to the death of massive stars [25,9] was strengthened in 2003, by the spectroscopic association of SN2003dh with GRB030329 [23, 17]. These massive progenitors have short lifetimes of $\sim 10^{7} \mathrm{yr}$, negligible over cosmological time scales, allowing LGRBs to be tracers of the cosmic star formation history (CSFH). It was seen that some LGRB samples had a high-redshift excess in comparison to the CSFH at high redshifts (e.g., [19]), suggesting that a non-linear dependence between the two existed. After initial studies of LGRB host galaxies, they were seen to be primarily low-metallic, low-mass, blue, and star forming galaxies [21, 15]. This apparent subset of galaxy type inspired many authors to apply it to the connection between the LGRB and CSFH [16]. However, metallicity is but one of many dependences investigated, along with: evolving LGRB luminosity functions (LF) [19], evolving stellar initial mass functions (IMF) [24], and sample selection effects [5], to name but a few. However, recent studies have begun to show a broader range of host galaxies, including both metal-rich [22,14] and massive-red galaxies [13]. Given recently acquired highly complete LGRB redshift samples [11, 19], the LGRB rate (LGRBR)-CSFH connection is reinvestigated over a range of galaxy properties.

\section{The sample}

The Gamma-Ray burst Optical Near-infrared (NIR) Detector (GROND) [12], a multi-channel imager mounted at the $2.2 \mathrm{~m} \mathrm{MPG/ESO}$ telescope at La Silla (Chile), has operated for the past 4 years as an automated GRB afterglow follow-up instrument. Due to the reduced attenuation from gas and dust at observer frame NIR wavelengths, GROND's multi-band capabilities in combination with its rapid-response obtains precise photometric redshifts when spectroscopic observations are not possible or fruitless (i.e., redshift desert, low signal-to-noise). This has facilitated, for the first time, highly complete GRB redshift samples. The GROND LGRB sample is created by selecting GRBs that: (i) are long duration ( $T_{90}^{\text {observer }}>2 \mathrm{~s}$ ) (ii) are detected by GROND $<4 \mathrm{hr}$ after the trigger and (iii) exhibit an X-ray afterglow. This results in a sample of 39 LGRBs: 31 spectroscopic redshifts, 6 photometric redshifts ( 3 of which are upper limits) and 2 with no optical/NIR afterglow detections and thus no redshift measurements. We will only consider the photometric sample which contains the spectroscopic and photometric redshifts, with a completeness of $\sim 90 \%$ (see Fig. 1).

\section{Modelling the long gamma-ray burst rate}

The following is a brief overview of the modelling, readers are encouraged to read [7] for the full description. The CSFH is simply the sum of all the star formation, $\operatorname{SFR}\left(M_{*}, z\right)$, of the contributing galaxies weighted by a galaxy mass function, $\phi\left(M_{*}, z\right)$, and can be described in the following way:

$$
\dot{\rho}=\int_{M_{1}}^{M_{2}} \zeta(z) \gamma\left(M_{*}, z, \varepsilon_{\mathrm{L}}\right) \operatorname{SFR}\left(M_{*}, z\right) \phi\left(M_{*}, z\right) \mathrm{d} M_{*}=\dot{\rho}\left(z, \varepsilon_{\mathrm{L}}, M_{1}, M_{2}\right) .
$$

where $M_{1}$ and $M_{2}$ are the upper and lower galaxy mass limits, and the $\operatorname{SFR}\left(M_{*}, z\right)$ has been taken from [1] and the $\phi\left(M_{*}, z\right)$ from [8]. The $\operatorname{SFR}\left(M_{*}, z\right)$ will still include massive galaxies that have ceased star formation, and so is corrected by $\zeta(z)$. We would like to investigate the contribution 

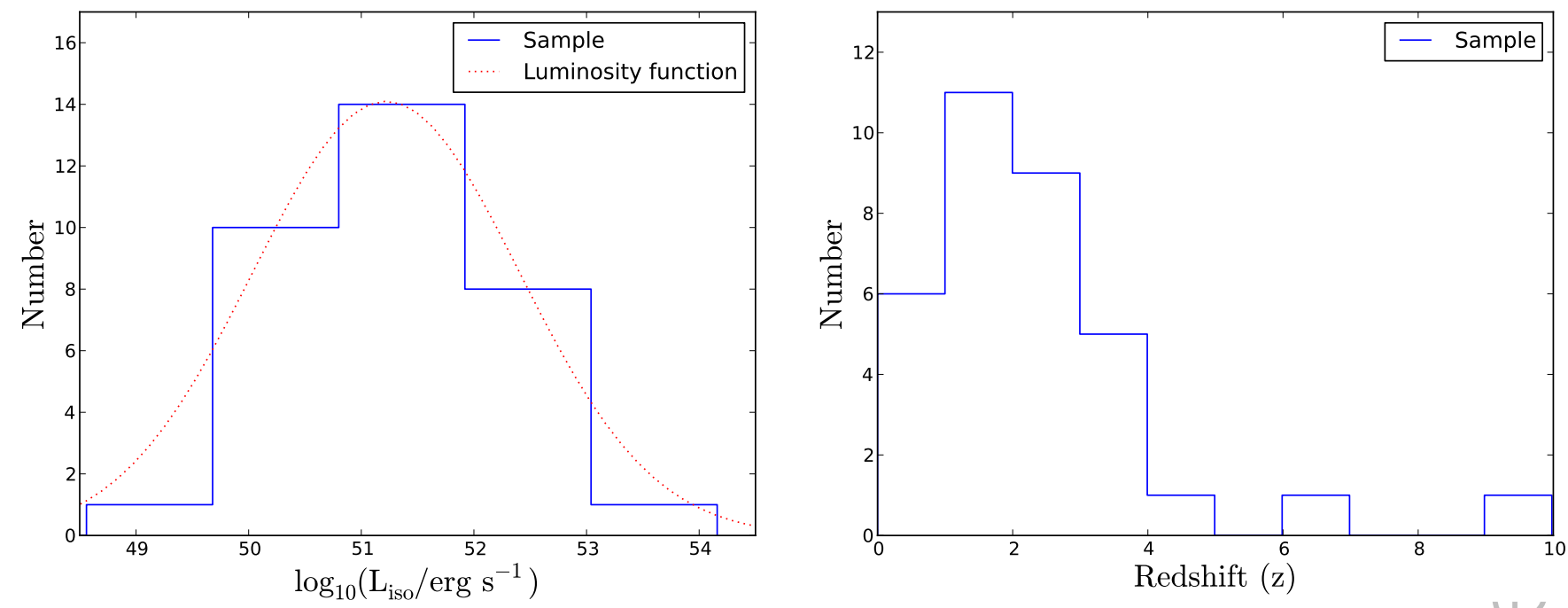

Figure 1: Left: The luminosity distribution of the GROND Photometric sample. The red-dotted line is the best fit LGRB luminosity function. Right: The redshift distribution of the GROND Photometric sample.
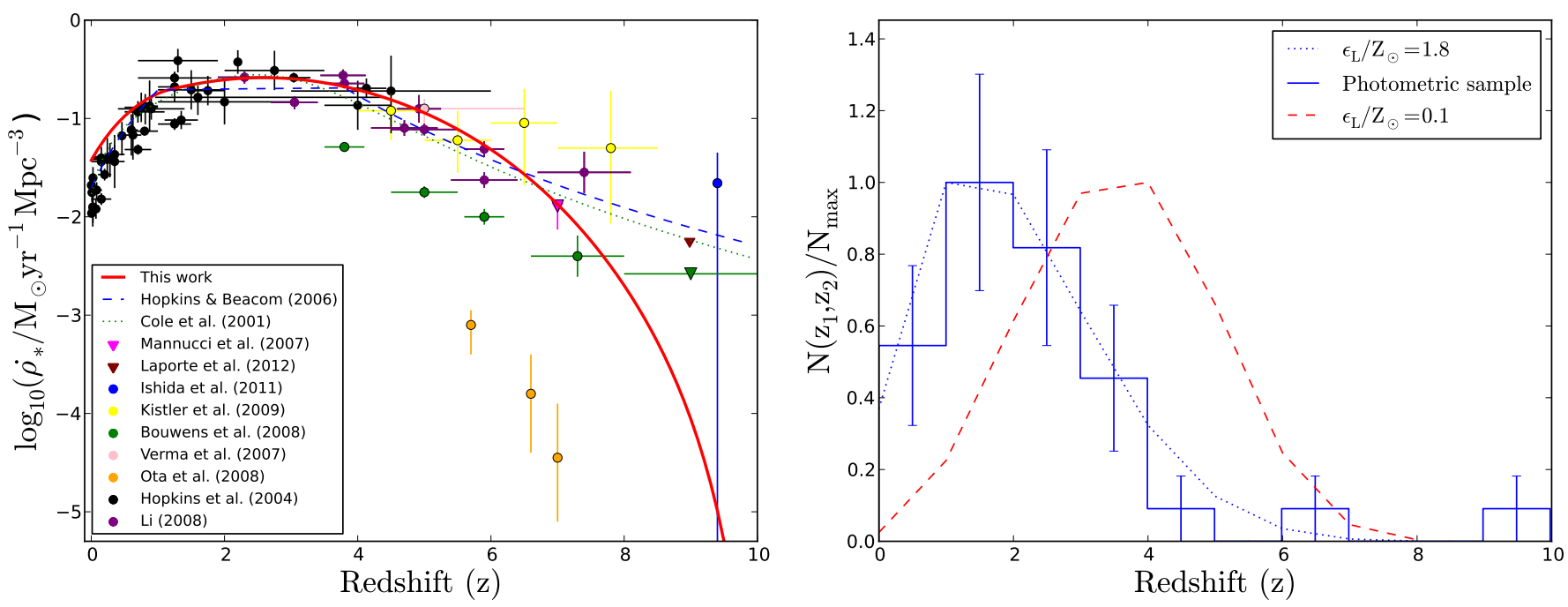

Figure 2: Left: The CSFH model described in Eqn. 3.1 is depicted by the red line, measured data are represented by black and purple dots, and corresponding parametric fits by the dashed and dashed-dotted lines. Right: The LGRBR model, both with metallicity cuts (red-dashed) and without (blue-dotted). They are compared to the GROND redshift distribution by the solid-blue lines.

to the CSFH solely from galaxies that lie below a specific metallicity cut, which is achieved by $\gamma\left(M_{*}, z, \varepsilon_{\mathrm{L}}\right)$. Both are simple step functions of the following type:

$$
\gamma\left(M_{*}, z, \varepsilon_{\mathrm{L}}\right)=\left\{\begin{array}{l}
1 \text { if } \varepsilon\left(M_{*}, z\right)<\varepsilon_{\mathrm{L}} \\
0 \text { if } \varepsilon\left(M_{*}, z\right) \geq \varepsilon_{\mathrm{L}}
\end{array} \text { and, } \zeta(\mathrm{z})=\left\{\begin{array}{ll}
1 & \text { if } M_{\mathrm{Q}}^{0}(1+z)^{3.5}>M_{*} \\
0 & \text { if } M_{\mathrm{Q}}^{0}(1+z)^{3.5} \leq M_{*}
\end{array},\right.\right.
$$



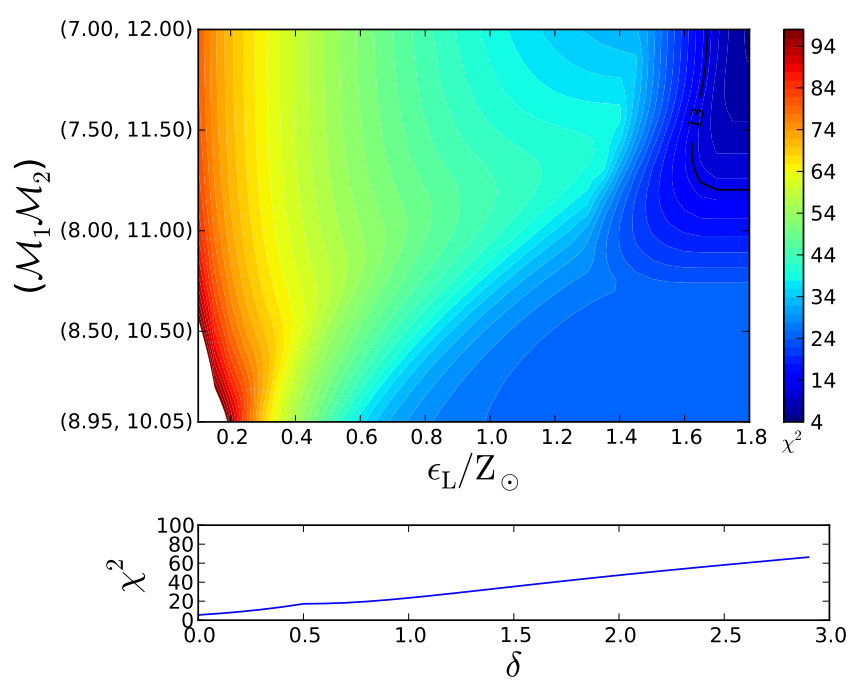

Figure 3: Upper Panel: The $\chi^{2}$ contour plot for $\delta=0$ (see lower panel) showing the best fit metallicity limits, $\left(\varepsilon_{L}\right)$, and mass ranges, $\left(\mathscr{M}_{1}=\log _{10}\left(M_{1}\right), \mathscr{M}_{2}=\right.$ $\left.\log _{10}\left(M_{2}\right)\right)$. The black line denotes the $1 \%$ acceptance level of the $\chi^{2}$ value, where blue is for better fits and red for worse fits. Lower Panel: The $\chi^{2}$ values for each $\delta$ value investigated. The resulting best-fit is for $\delta=0$ and so only the contour plot for this value is depicted in the upper panel.

where $\varepsilon\left(M_{*}, z\right)$ is the Savaglio relation [20], $\varepsilon_{\mathrm{L}}$ is the metallicity cut-off, and $M_{Q}$ is the local quenching mass $[2,10]$. It is then possible to obtain a modelled LGRB number between two redshifts $z_{1}$ and $z_{2}$ using the following relation:

$$
N\left(z_{1}, z_{2}\right)=\eta_{\operatorname{grb}} \frac{\int_{M_{\mathrm{BH}}}^{M_{\max }} \psi(m) \mathrm{d} m}{\int_{M_{\min }}^{M_{\max }} m \psi(m) \mathrm{d} m} \int_{z_{1}}^{z_{2}} \frac{\dot{\rho}\left(z, \varepsilon_{L}, M_{1}, M_{2}\right)(1+z)^{\delta}}{1+z} \frac{\mathrm{d} V}{\mathrm{~d} z} \int_{L_{\mathrm{limit}(z)}}^{\infty} \phi(L) \mathrm{d} L \mathrm{~d} z,
$$

where $\phi(L)$ is the LGRB luminosity function, $\delta$ is the evolution dependence, $\frac{\mathrm{d} V}{\mathrm{~d} z}$ is the co-moving volume, $\frac{1}{(1+z)}$ is the cosmological time dilation, $\psi(m)$ is the Salpeter IMF [18] with upper and lower mass limits of $\mathrm{M}_{\text {low }}$ and $\mathrm{M}_{\text {up }}$ respectively, $\mathrm{M}_{\mathrm{BH}}$ is the minimum mass that forms a black hole, $L_{\text {limit }}(z)$ is the instrumental luminosity limit, and $\eta_{\text {grb }}$ is the LGRB probability. The luminosity limit, $L_{\text {limit }(z)}$, of the sample can be calculated using the luminosity distance, $D_{L}$, of the form $L_{\text {limit }}=$ $4 \pi D_{L}^{2} F_{\text {limit }}$. By taking $F_{\text {limit }}$ to be the lowest luminosity of the sample, results in the following flux limit: $F_{\text {limit }}=1.08 \cdot 10^{-8} \mathrm{erg} \mathrm{s}^{-1} \mathrm{~cm}^{-2}$. The LGRB probability contains extra factors that convert the CSFH in to a LGRBR and is described easily by:

$$
\eta_{\text {grb }}=\Delta T \Delta \Omega \times \eta_{\text {time }} \eta_{\mathrm{X}-\text { ray }} \eta_{\text {redshift }} \times n_{\mathrm{LF}}\left(1-\cos \theta_{\text {jet }}\right),
$$

where $\Delta T$, and $\Delta \Omega$, are the survey time length and fractional area covered by the instrument respectively. $\eta_{\text {time }}, \eta_{\mathrm{X}-\text { ray }}$, and $\eta_{\text {redshift }}$ are the biases introduced into the chosen sample: fraction observed $<T \mathrm{hr}$ after the trigger, fraction with an X-ray afterglow, and redshift completeness, respectively. Finally, $n_{\mathrm{LF}}$, is the normalisation of the LGRBLF, and $\theta_{\text {jet }}$ is the collimation angle of the jet. The resulting model can be seen in Fig. 2. All the input values can be found in [7].

\section{Grid approach and resulting galaxy properties}

The 3-dimensional parameter space for the models derived in Sec. 3 contains: metallicity $\left(\varepsilon_{L}\right)$ and mass range $\left(\mathscr{M}_{1}, \mathscr{M}_{2}\right)$ constraints on the galaxies contributing to the CSFH, and an evolution dependence $(\delta)$ between the LGRBR and the CSFH. To find the best set of parameters, a grid-like approach is utilised. This consists of calculating a LGRBR for each combination of parameters 


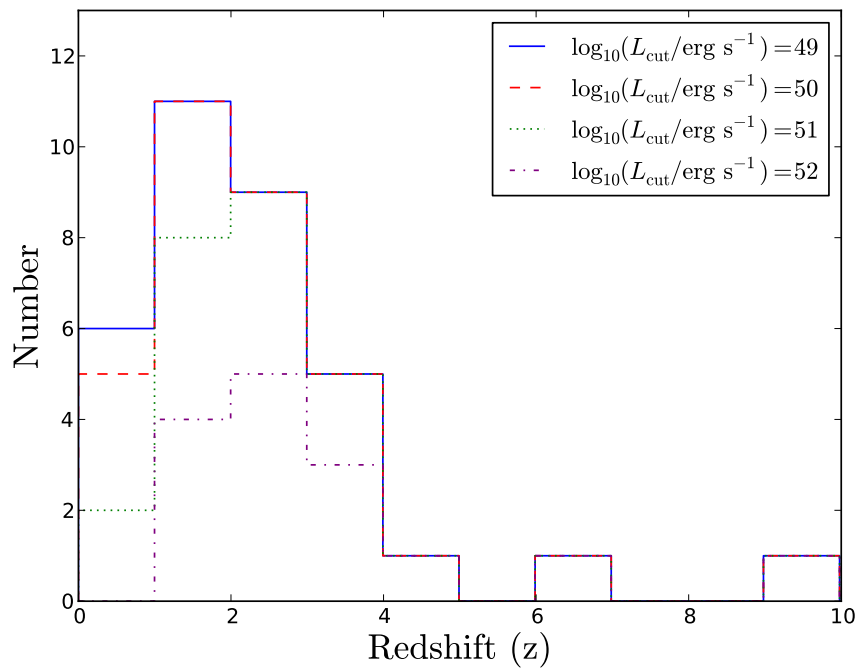

Figure 4: The resulting GROND Photometric sample after a luminosity threshold has been applied. The peak of the distribution is seen to shift to much higher redshifts.

and comparing the resulting distribution to the GROND sample (Sec. 2) utilising $\chi^{2}$ analysis (seen pictorially in Fig. 2). The parameter range investigated is $\varepsilon_{\mathrm{L}} / \mathrm{Z}_{\odot}=0.1-1.8$ with steps of 0.1 , $\mathrm{M}_{1} / \mathrm{M}_{\odot}, \mathrm{M}_{2} / \mathrm{M}_{\odot}=\left(10^{7}, 10^{12}\right) \ldots\left(10^{7+\Delta x}, 10^{12-\Delta x}\right)$ for steps of $x=0.05$, and $\delta=0-2.9$ with steps of 0.1 . The evolution parameter of $\delta<0$ could have been investigated, but due to the unphysical nature in terms of the collapsar model it was left for future studies. The resulting best-fit contour plot of $\delta=0$ can be seen in Fig. 3. This shows that the best fit CSFH is one that does not require any constraints on the galaxy types contributing (e.g., metallicity or mass ranges), nor is there any evolution dependence between the CSFH and LGRBR. This would imply that LGRBs are not selecting specific types of galaxy and are tracing areas of star formation (i.e., LGRBR CSFH). These implications could be altered depending on the model types discussed in the previous section.

\section{Discussion}

There are many possible avenues one could take when creating the model of the LGRBR (Sec. 3), and by making different choices the results, in some non-trivial way, could change. The main three possibilities are: (i) the LGRBLF, (ii) the stellar IMF and (iii) the sample selection criteria.

(i) The GRBLF can be of different forms; power-law, log-normal, redshift-evolving, and even an evolving luminosity limit.

(ii) The stellar IMF can also be of different type, e.g. Salpeter [18], Chabrier [4] and even redshift evolving (Davé) [6].

(iii) If completeness levels of a sample are increased using luminosity cuts, it can introduce extra biases if the redshifts were not obtained in a non-biased way, for example, shifting the peak of the LGRB redshift distribution to higher redshifts, requiring there to be biases introduced (see Fig. 4).

\section{References}

[1] N. Bouché, A. Dekel, R. Genzel, The Impact of Cold Gas Accretion Above a Mass Floor on Galaxy Scaling Relations, ApJ 718 (2010) 1001 [astro-ph:0912.1858].

[2] K. Bundy, R. S. Ellis, C. J. Conselice et al., The Mass Assembly History of Field Galaxies: Detection of an Evolving Mass Limit for Star-Forming Galaxies, ApJ 651 (2006) 120 [astro-ph/ 0512465]. 
[3] X. -F. Cao, Y. -W. Yu, K. S. Cheng, X. -P. Zheng, The luminosity function of Swift long gamma-ray bursts, MNRAS 416 (2011) 2174 [a stro-ph/1101 . 0866].

[4] G. Chabrier, Galactic Stellar and Substellar Initial Mass Function, PASP 115 (2003) 763 [astro-ph/0304382].

[5] D. M. Coward, D. Guetta, R. R. Burman, Where are the missing gamma-ray burst redshifts?, MNRAS 386 (2008) 111 [astro-ph/ 0711.0242 ].

[6] R. Davé, B. D. Oppenheimer, and S. Sivanandam, Enrichment and pre-heating in intragroup gas from galactic outflows, MNRAS 391 (2008) 110 [astro-ph/0710 . 0381].

[7] J. Elliott, J. Greiner, S. Khochfar et al., The long $\gamma$-ray burst rate and the correlation with host galaxy properties, A\&A 539 (2012) 113E [astro-ph/1202 . 1225].

[8] A. Fontana, S. Salimbeni, A. Grazian et al., The Galaxy mass function up to $z=4$ in the GOODS-MUSIC sample: into the epoch of formation of massive galaxies, A\&A 459 (2006) 745 [astro-ph/0609068].

[9] T. J. Galama, P. M. Vreeswijk, J. van Paradijs, et al., An unusual supernova in the error box of the $\gamma$-ray burst of 25 April 1998, Nature 395 (1998) 670 [a st ro-ph/9806175].

[10] V. Villar, J. Gallego, P. Pérez-González, et al. Star Formation Rates and Stellar Masses of H-alpha Selected Star-Forming Galaxies at z=0.84: A Quantification of the Downsizing, ApJ, 740 (2011) 47 [astro-ph/1107.4371].

[11] J. Greiner, T., Krühler, S., Klose et al., The nature of “dark” gamma-ray bursts, A\&A 526 (2011) A30 [astro-ph/1011.0618].

[12] J. Greiner, W. Bornemann, C. Clemens et al., GROND-a 7-Channel Imager, PASP 120 (2008) 405 [astro-ph/0801.4801].

[13] T. Krühler, J. Greiner, P. Schady et al., The SEDs and host galaxies of the dustiest GRB afterglows, A\&A 534 (2011) A108 [astro-ph/1108.0674].

[14] T. Krühler, J. P. U. Fynbo, S. Geier, et al., The metal-enriched host of an energetic gamma-ray burst at $z \sim 1.6$ (2012) [astro-ph/1203.1919].

[15] E. Le Floc'h, P. -A. Duc, I. F. Mirabel et al., Are the hosts of gamma-ray bursts sub-luminous and blue galaxies?, A\&A 400 (2003) 499 [astro-ph/astro-ph/ 0301149 ].

[16] L. -X. Li, Star formation history up to $z=7.4$ : implications for gamma-ray bursts and cosmic metallicity evolution, MNRAS 388 (2008) 1487 [astro-ph/ 0710 . 3587]

[17] T. Matheson, P. M. Garnavich, K. Z. Stanek et al., Photometry and Spectroscopy of GRB 030329 and Its Associated Supernova 2003dh: The First Two Months, ApJ 599 (2003) 394 [astro-ph/0307435].

[18] E. Salpeter, The Luminosity Function and Stellar Evolution, ApJ 121 (1995) 161.

[19] R. Salvaterra, S. Campana, S. D. Vergani et al., A Complete Sample of Bright Swift Long Gamma-Ray Bursts. I. Sample Presentation, Luminosity Function and Evolution, ApJ 749 (2011) 68 [astro-ph/1112.1700].

[20] S. Savaglio, K. Glazebrook, D. Le Borgne et al., The Gemini Deep Deep Survey. VII. The Redshift Evolution of the Mass-Metallicity Relation, ApJ 635 (2005) 260 [astro-ph/astro-ph/0508407].

[21] S. Savaglio, K. Glazebrook, D. Le Borgne, The Galaxy Population Hosting Gamma-Ray Bursts, ApJ 691 (2009) 182 [astro-ph/0803.2718].

[22] S. Savaglio, A. Rau, J. Greiner, et al., Supersolar metal abundances in two galaxies at $z \sim 3.57$ revealed by the GRB 090323 afterglow spectrum, MNRAS 420 (2012) 627 [astro-ph/1110.4642].

[23] K. Z. Stanek, T. Matheson, P. M. Garnavich et al., Spectroscopic Discovery of the Supernova 2003dh Associated with GRB 030329, ApJ 591L (2003) 17 [astro-ph/ 0304173 ]. 
[24] F. Y. Wang and Z. G. Dai, An Evolving Stellar Initial Mass Function and the Gamma-ray Burst Redshift Distribution, ApJ 727 (2011) 34 [a stro-ph/1101.3633].

[25] S. E. Woosley, Gamma-ray bursts from stellar mass accretion disks around black holes, ApJ 405 (1993) 273 . 